\title{
Conception, COVID, and Communication
}

\author{
Graeme T. Laurie ${ }^{1}$
}

Published online: 14 April 2021

() National University of Singapore and Springer Nature Singapore Pte Ltd. 2021

The June 2021 issue of the Asian Bioethics Review picks up where the March 2021 Special Issue left off. This last issue explored the extensive interconnections between reproductive practices, populations politics, and bioethics, emphasising how many of the issues are brought into even more acute focus in light of the COVID-19 pandemic. The current issue continues to explore these twin strands of reproductive ethics and pandemic politics, albeit not as overtly connected as some of the excellent discussions found in the Special Issue. But this simply highlights all the more clearly our on-going responsibility to uncover and examine yet further the underlying influences and dynamics that impact the range of topics that are brought under the umbrella of bioethics.

In the opening paper of this issue, Nisha [https://doi.org/10.1007/s41649-02100169-z] examines diverse feminist responses to the growing involvement of technology with human reproduction, in particular the effects on women's choices, on the identity of the maternal self, and on roles and responsibilities. Engagement with the literature suggests a polarisation of views and reactions to the increased involvement of technology, and an egalitarian model of engagement with technology is proposed as a possible way through the debates. Alsomali and Hussein [https://doi.org/10.1007/s41649-021-00167-1] focus on a particular example of novel technological development in reproduction-CRISPR-Cas9-to examine the ethics of this new technology from both regulatory and religious perspectives. Arguing from the first principles of Islamic law (Maqasid al Shari'a) and related maxims (Qawaid Fiqhiyyah), their article posits that a defensible case for this technology can be made. From this, the authors then explore how this perspective can be used to inform future regulation, especially against the background of examples of unregulated use that have emerged to date. Our third Original Article relating to reproduction strengthens the contribution of this journal in the realm of empirical research. Kuek et al. [https://doi.org/10.1007/s41649-021-00166-2] offer findings relating to attitudes towards the phenomenon of saviour siblings made possible by technological developments, and they set their discussion against the backdrop that much of the current literature on the ethics of this practice reflects a Western liberal

Graeme T. Laurie

graeme.laurie@ed.ac.uk

1 Edinburgh Law School, The University of Edinburgh, Edinburgh, UK 
perspective. Important research questions therefore arise for the Asia region, and the authors focus on attitudes in Malaysia where there is no specific legal framework for reproductive technologies. In many senses, then, this country stands at a threshold in asking itself about whether, how, and when to regulate ARTs in general, and the technologies related to saviour siblings in particular. Stakeholder insights therefore become especially valuable and the authors report the findings from their empirical work in this regard. These findings show general support and also reveal some concerns not widely discussed elsewhere, such as the economic implications involved. This is an excellent example of how bioethically informed empirical research can inform future policy and practice.

The article offered by Ong et al. [https://doi.org/10.1007/s41649-021-00165-3] is a further illustration of the potential value of empirical research. In this piece, the authors report on a qualitative study of focus group participants in Singapore invited to discuss perceptions of 'precision' and 'personalised' medicine. In particular, the work focusses on the role and importance of clear communication practices and strategies for engaging with publics on the introduction and use of such new techniques, as well as on the wider implications such as the need for extensive data analytics. As with the example from Malaysia, the findings here are of particular importance for informing policy, and in this case in pursuing the all-important social licence that is required for the introduction of any new technology into our lives with consequences for our health and well-being.

The second strand of papers in this issue relates to ethical responses to the COVID-19 pandemic. As such, they represent the end of our highly successful call for papers launched in 2020. The journal received almost 60 submissions on many bioethical topics related to the pandemic, of which currently 25 are accepted for publication. The formal call is now closed, but the journal remains disposed to receiving original submissions that are related to COVID-19 and which do not overlap with content already published in the journal. A priority remains to publish accounts concerning the experience of countries in responding to the pandemic, particularly in the Asia-Pacific region.

The COVID-19 papers in this issue reflect the approach that we have adopted. Thus, Kraaijeveld [https://doi.org/10.1007/s41649-020-00154-y] engages with the highly controversial practice of "lockdown"-in all the forms that this has taken around the globe - arguing against this practice and in favour of an altruistic approach that, in the author's view, better preserves freedoms, has a greater chance of avoiding potential injustices, and can obviate a sense of being lost or powerless during these difficult times.

Our ambition to capture countries' experiences of dealing with COVID-19 is further realised by the papers from Lee and Kang [https://doi.org/10.1007/ s41649-021-00164-4] and Hettiarachchi et al. [https://doi.org/10.1007/s41649020-00153-z], respectively examining the contexts of South Korea and Sri Lanka. As to the former, the authors challenge to a large extent the external perspective that South Korea's response has been an unproblematic 'success'; instead, they offer a compelling analysis of the actions and motivation of various groups within the country that have acted in very opposite and oppositional ways with respect to the pandemic and their support of, or rejection of, governmental policy. This 
reveals important new insights into the potential underlying dynamics at work in trying to influence and drive social behaviour in the name of sound ethical responses to a public health crisis such as COVID-19. As to the latter country, living in Sri Lanka, this Editor can speak from personal experience about how 'hard' the initial lockdown was-it was tantamount to a full curfew through late March 2020 until early May 2020. This raises important challenges of having meaningful conversations about what "lockdown" across the globe has actually meant, because countries have taken such varied approaches. Hettiarachchi et al. [https://doi.org/10.1007/s41649-020-00153-z] offer commentary on one of the most draconian responses, in Sri Lanka, and their insights are valuable not only with respect to what was done in the country but also regarding lessons learned and (relative) freedoms earned as a result.

The contribution from Lyngdoh [https://doi.org/10.1007/s41649-021-00168-0] brings this issue neatly back to the realm of philosophy; it can be seen as something of meta-commentary on all that has gone before in the COVID-19 articles that we have published to date. It focuses on the possibility/impossibility of community thinking, particularly in India. Not only does it remind us of the rich contributions from philosophy on the value and importance of engaging with the Other but it also shows that individual difference and togetherness in crisis need not be seen in inevitable conflict. This message, perhaps more than most, is particularly pertinent in the times of COVID-19.

Our final paper on COVID-19 is a further example of the importance of our Student Voices section. Ali et al. [https://doi.org/10.1007/s41649-020-00155-x] tackle the topic of misinformation during the pandemic, driven by the self-evident increase of the role of social media in people's lives as they experienced lockdowns of varying degrees of severity. Drawing directly on the experiences of fellow students, the authors highlight the myriad ways in which misinformation can drive numerous unethical outcomes and injustices, not to speak of the adverse effects on health workers at the forefront of dealing with the pandemic. Helpful suggestions are made about how to monitor and evaluate this phenomenon, including how to mitigate the worst vagaries of its impact.

A sub-theme of this issue has been the importance of meaningful communication. Thus, Ong et al. [https://doi.org/10.1007/s41649-021-00165-3] have explored various mechanisms and routes to productive engagement in the context of precision medicine, while Ali et al. [https://doi.org/10.1007/s41649020-00155-x] have exposed the serious ethical risks related to misinformation, whether negligent, reckless, or deliberate. But in-between, there is also the crucially important cultural question of the language medium in which healthrelated communication itself takes place. Jayasinghe [https://doi.org/10.1007/ s41649-021-00172-4] offers the example of Sri Lanka as a former colony of Britain and where once English was the official language of all formal communication. Albeit that this has been replaced by Sinhala and Tamil, English remains the principal medium of communication in healthcare. Through an historical account and understanding of language policies and practices, the author amply demonstrates how this leads to current day discrimination and marginalisation for many Sri Lankans and a real barrier to health literacy. This illustrates one particular 
example of the enduring invidious impact of colonisation, and it is a matter to which this journal is committed to return in the future.

Publisher's Note Springer Nature remains neutral with regard to jurisdictional claims in published maps and institutional affiliations. 